\title{
THE CALIFORNIA BIOMONITORING PROGRAM: PERSISTENT ORGANIC POLLUTANTS IN ARCHIVED AND CONTEMPORARY SERUM
}

\author{
M. PETREAS* \\ J.S. PARK \\ M. WANG \\ Y. WANG \\ W. GUO \\ D. TARRANT \\ A. RHEE \\ S. HARWANI
}

Received: 20/12/11

Accepted: 31/01/12

\author{
Environmental Chemistry Laboratory \\ California Department of Toxic Substances Contro, \\ 700 Heinz Street, Berkeley, CA 94710, USA
}

*to whom all correspondence should be addressed: e-mail: mpetreas@dtsc.ca.gov

\section{ABSTRACT}

In 2006, California passed legislation establishing the first State Biomonitoring Program in the USA. The main goals are to: 1) Determine levels of environmental chemical contaminants in a representative sample of Californians; 2) Establish trends in the levels of these chemicals over time; 3) Assess the effectiveness of public health efforts and regulatory programs to decrease exposures to specific chemicals.

As part of the Biomonitoring Program, our laboratory will be conducting analyses for Persistent Organic Pollutants (POPs) such as organochlorine pesticides (OCPs), PCBs, polybrominated diphenyl ethers (PBDEs), perfluorinated chemicals (PFCs), triclosan, phenols, OH-PCBs and $\mathrm{OH}-$ PBDEs. Prior to this program, we had conducted a number of epidemiologic studies using specimens collected from the 1960s to the present and analysing them for POPs. Serum, milk and adipose samples were extracted and the neutral fractions were cleaned up using deactivated Florisil column chromatography, and analyzed for PCBs, OCPs and PBDEs by dual column GC-ECD and/or High Resolution Mass Spectrometry. Online Solid Phase Extraction - HPLC- Turbo ion Spray Tandem Mass Spectrometry was used to analyze PFCs. Following standard conventions, results are expressed on a lipid basis (PCBs, PBDEs, OCPs), or on a volume basis (PFCs, Triclosan, Phenols, $\mathrm{OH}-\mathrm{PCBs}, \mathrm{OH}-\mathrm{PBDEs}$ ). A Quality Management system tracks all laboratory work.

We had first reported the absence of PBDEs in serum samples from 1960s California populations as opposed to their presence in samples collected in the 1990s. We confirmed this observation with the analysis of over 1500 samples from the 1960s. In contemporary serum, the abundance of PBDE congeners was in the order of BDE-47>153>99>100, while BDE-209 was measurable in only a few of the samples. We could trace the increase of PFOA from the1960s to the 1980s, followed by a slight decrease in 2009. On the other hand, PFOS and PFHxS were highest in the 1960s, with similar decreasing trends from the1980s to 2009.

In addition to addressing the research hypotheses of each epidemiologic study, data compiled across studies can show trends such as the emergence of PBDEs, and the decline in PCBs, phenols, OCPs and some PFCs over time. In addition, determinants of exposures (age, country of birth, ethnicity and reproductive history) can be identified, allowing for optimal sampling designs to account for the population diversity in California and can also be used in questionnaires to assess exposures. These data help establish a baseline before the new Biomonitoring Program launches its state-wide surveys.

KEYWORDS: Biomonitoring, California, OCPs, PBDEs, PCBs, PFCs, POPs, serum. 


\section{INTRODUCTION}

A multitude of environmental chemicals may be toxic to humans, but with few exceptions, relatively little is known about the presence and disposition of these chemicals inside peoples' bodies. Some chronic diseases have been linked to exposures to synthetic chemicals, including a variety of common chemicals that can mimic or block the actions of hormones necessary for growth and the maintenance of health. Increases in thyroid disease, breast and brain cancer, infertility, asthma, autism and other developmental diseases have heightened public concern about potential effects of environmental exposures.

In the USA, the Centers for Disease Control recently released its Fourth National Report on Human Exposure to Environmental Chemicals, containing data on chemicals in blood and urine samples from participants in the National Health and Nutrition Examination Survey (NHANES). The CDC data show elevated levels of some contaminants throughout the U.S., but also demonstrate that cotinine, a biological marker of exposure to environmental tobacco smoke, has declined nationally due to aggressive anti-smoking campaigns. Such surveys are excellent indicators of the efficacy of public health interventions. However, NHANES is designed to be representative of the overall U.S. population, not individual states. Given the lack of state-specific data from NHANES and the diversity of California's population, particularly the large number of immigrants whose dietary and other environmental exposures are likely to differ from "mainstream" population patterns, it was appropriate that California initiate its own biomonitoring program, designed to include both measurements of contaminants in body fluids and an assessment of potential routes of exposure. The latter will help design interventions and gauge the efficacy of efforts by regulatory and Public Health agencies.

Biomonitoring can provide estimates of how many people in California have significant levels of these chemicals or their metabolites in their bodies, which, when combined with toxicity data, can provide a sense of some potential health risks to California's population. Biomonitoring data can also help identify sources of exposure to toxic chemicals. This information can be used in follow-up studies to determine whether specific individuals or groups have unusually high exposures to these contaminants due to their diets, occupations, places of residence, or other factors. Ultimately, this information may lead to new insights into links between environmental contaminants and chronic diseases.

When fully implemented, the California Biomonitoring Program (CECBP) will provide a scientifically rigorous approach to biomonitoring, resulting in the following principal outcomes:

1) Determine levels of environmental chemical contaminants in a representative sample of Californians;

2) Establish trends in the levels of these chemicals over time;

3) Assess the effectiveness of public health efforts and regulatory programs to decrease exposures to specific chemicals.

Prior to this new Biomonitoring Program, our laboratory had conducted a number of epidemiologic studies using blood, milk or adipose collected from the 1960s to the present. Samples were analysed for Persistent Organic Pollutants (POPs) such as organochlorine pesticides (OCPs), PCBs, polybrominated diphenyl ethers (PBDEs), perfluorinated chemicals (PFCs), triclosan, phenols, $\mathrm{OH}-$ $\mathrm{PCBs}$ and $\mathrm{OH}-\mathrm{PBDEs}$. Following standard conventions, results are expressed on a lipid basis (PCBs, PBDEs, OCPs), or on a serum volume basis (PFCs, Triclosan, Phenols, OH-PCBs, OHPBDEs). A Quality Management system tracks all laboratory work.

In addition to addressing the research hypotheses of each epidemiologic study, data compiled across studies can show trends such as the emergence of PBDEs, and the decline in PCBs, phenols, OCPs over time. Moreover, significant determinants of exposures (age, country of birth, ethnicity and reproductive history) can be identified. This information would allow for optimal sampling designs to account for the population diversity in California and can also be used in questionnaires to assess exposures. These data help establish a baseline of chemical contaminants before the new Biomonitoring Program launches its statewide surveys. 


\section{MATERIALS AND METHODS}

\section{Populations Studied}

We only focus on studies of women (recruited from studies on reproductive effects, breast cancer and healthy adults) to facilitate comparisons. All subjects had given written informed consent at the time of recruitment. Samples had been kept at $-20^{\circ} \mathrm{C}$ or $-80^{\circ} \mathrm{C}$ and were analyzed by our laboratory using similar methods. Five distinct time periods of recruitment are examined:

1960s:

D Serum samples (Petreas et al., 2011) from the California Health and Development Studies, a longitudinal birth cohort of over 20,000 births in Northern California, recruiting pregnant women between 1959 and 1967. ( $\mathrm{N}=1,200)$.

$1980 \mathrm{~s}$

$>$ Serum samples from cancer-free women (controls) participating in a case-control study of breast cancer in the San Francisco area (preliminary $\mathrm{N}=30$ ).

1996-98:

Adipose samples (Petreas et al., 2003) from women participating in a breast cancer study in the San Francisco area $(\mathrm{N}=162)$.

$>$ Serum samples from Laotian immigrants to the San Francisco area participating in a study of ovarian function (Windham et al., 2005) ( $\mathrm{N}=50)$.

2003-05:

Milk samples (Hooper et al., 2007) from a community-based California study ( $\mathrm{N}=82)$.

2008-09:

$>$ Serum samples from adult California women from a pilot study (preliminary $N=30$ ).

$>$ Serum samples from a pilot study on pregnant women (Zota et al., 2010) (preliminary N=25).

Analytical Methods: Our methods for multiresidue analyses for the simultaneous extraction of PCBs, organochlorine pesticides, PBDEs, select new BFRs and PFCs in serum, milk and adipose have been described in detail (Rogers et al., 2004; Brown et al.,2007; She et al., 2007; Wang et al., 2010)

Serum: Serum samples were extracted and the neutral fractions were cleaned up using deactivated Florisil column chromatography, they were analyzed for PCBs, organochlorine pesticides and PBDE 47, 99, 100, 153 and 209 on a Varian 3800 GC-ECD equipped with RTX-5MS capillary column (60m $\times 0.25 \mathrm{~mm}$ i.d., 0.25 um thickness, $)$ and DB-XLB capillary column $(60 \mathrm{~m} \times 0.25 \mathrm{~mm}$ i.d., $0.25 \mathrm{um}$ thickness). Injection (2 uL) was made in split/splitless mode. PBDEs analyzed by ECD were confirmed by high resolution mass spectrometry. Pooled serum samples were used as in-house control samples as described elsewhere (Rogers et al., 2004; Brown et al.,2007; She et al., 2007; Wang et al., 2010). Online Solid Phase Extraction - High Performance Liquid Chromatography Turbo ion Spray - Tandem Mass Spectrometry was used to analyze 12 PFCs in serum samples (Wang et al., 2010). Briefly, $100 \mathrm{uL}$ of serum sample is diluted with formic acid and injected into the online SPE-HPLC-TIS-MS/MS system. The analytes of interest are first purified and concentrated in the SPE column (C18), then separated by a C8 HPLC column before entering the MS/MS system operating in negative-ion spray ionization mode.

Adipose: Adipose samples were spiked with ${ }^{13}$ C-labeled internal standards, cleaned up with Gel Permeation Chromatography (GPC) and Florisil column; the extracts were reduced to $10 \mu \mathrm{L}$ and recovery standards added. Analysis was carried out on a Varian $3800 \mathrm{GC}$ with a deactivated injection port liner (Siltek, $4.0 \mathrm{~mm}$ id, with glass frit) in splitless mode, with no pressure pulse and a Varian VF-5ms column ( $30 \mathrm{~m} \times 0.25 \mathrm{~mm}$ id $\times 0.25 \mu \mathrm{m})$. A $1200 \mathrm{~L}$ mass spectrometer (Varian) was operated in extended dynamic range mode using electron impact ionization and MS/MS detection (Brown et al., 2007). 
Milk: Milk samples were lyophilized, extracted using an ASE 200TM, cleaned up by mixed silica gel column, and GPC. The mixtures were spiked with ${ }^{13} \mathrm{C}$ internal standards of PBDEs and PCBs and analyzed on a Hewlett-Packard 6890 gas chromatograph, with a split/splitless injector and a DB-5 column $(60 \mathrm{~m}, 0.25 \mathrm{~mm}$ ID, $0.25 \mathrm{um})$. A Finnigan MAT95 high-resolution mass spectrometer, operated in electron impact mode at 9000 resolution for PCBs and 6000 for PBDEs (She et al., 2007).

\section{RESULTS AND DISCUSSION}

We had first reported the absence of PBDEs in serum samples from 1960s California populations as opposed to their presence in samples collected in the late 1990s (Petreas et al., 2003). We confirmed this observation with the analysis of over 1200 serum samples from the $1960 \mathrm{~s}$. The abundance of PBDE congeners was in the order of BDE-47>153>99>100, while BDE-209 was measurable in only a few of the contemporary serum samples. Some of the new BFRs (e.g., ATE, $\alpha, \beta-T B E C H$, PBT, PBEB, HBB, DPTE, DBDPE) were not detected in contemporary serum, or detectable but at levels below quantitation. Analyses are underway for additional BFR replacements of PBDEs.

PBDE levels (BDE 47 shown in Fig 1) in California samples exceed levels in the US population (NHANES, 2003-04) (Sjodin et al., 2008). As expected, younger women (participants in reproductive studies) have higher levels than older women (participants in breast cancer studies, pilot study). With our limited preliminary data set it is not clear whether PBDEs have peaked in the mid 2000s and started declining, possibly as a result of the ban. A clear difference appears between samples from pregnant and younger women and those from older women. This may be a function of age or of changes during pregnancy; additional data are being generated to test that hypothesis.

In contrast to PBDEs, levels of legacy contaminants such as PCBs and chlorinated pesticides in California women (serum, adipose, milk), along with the PFCs (Wang et al., 2010), showed declining temporal changes over the past 50 years (Fig 2 and Fig 3 ).

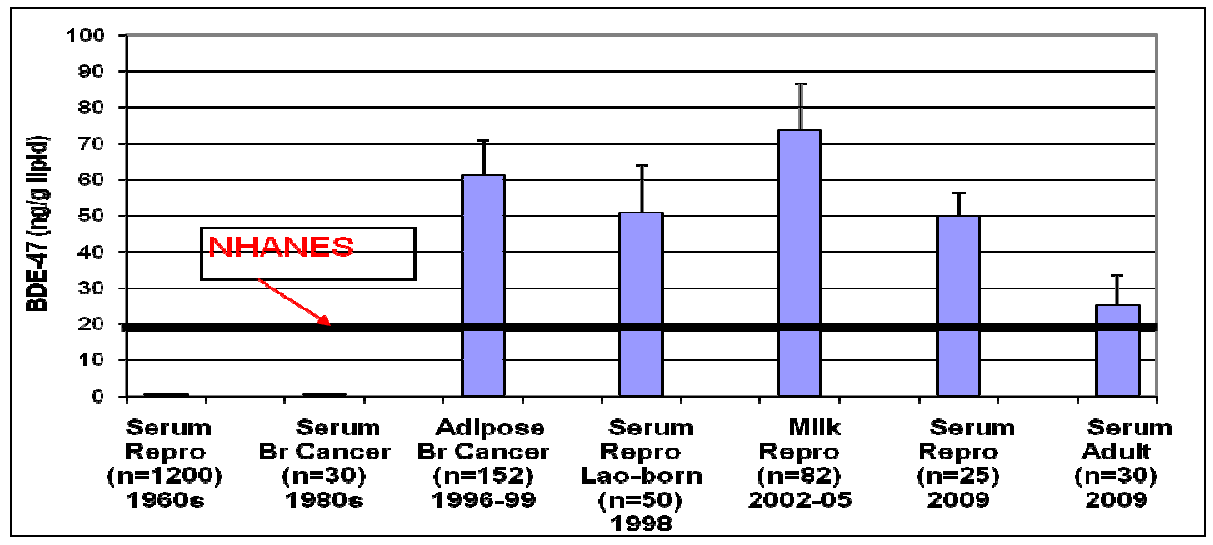

Figure 1. BDE-47 in California Women

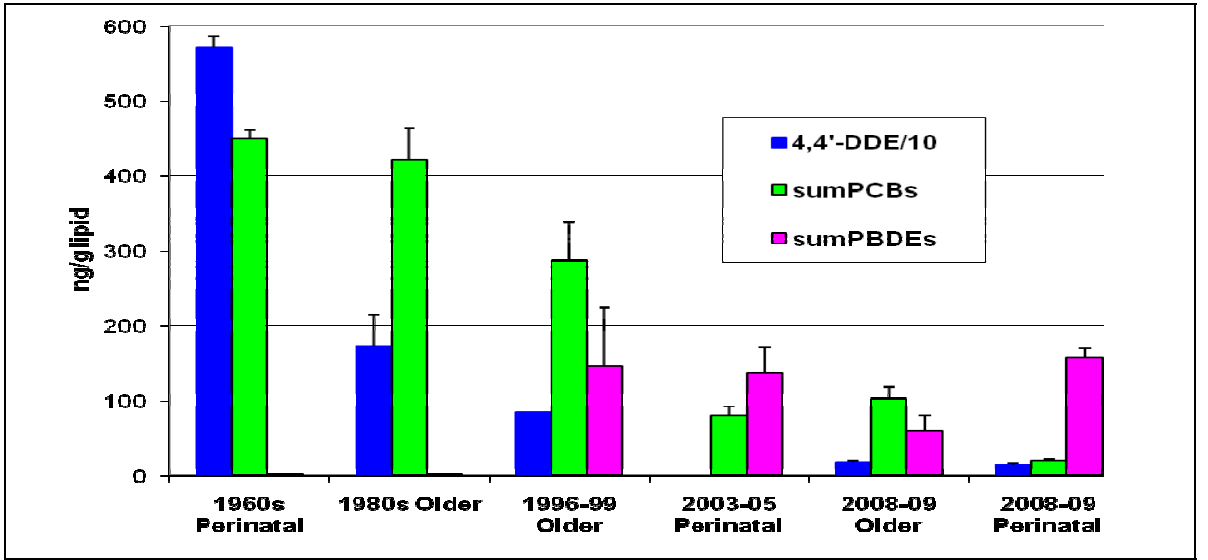

Figure 2. Temporal Trends in California Women 


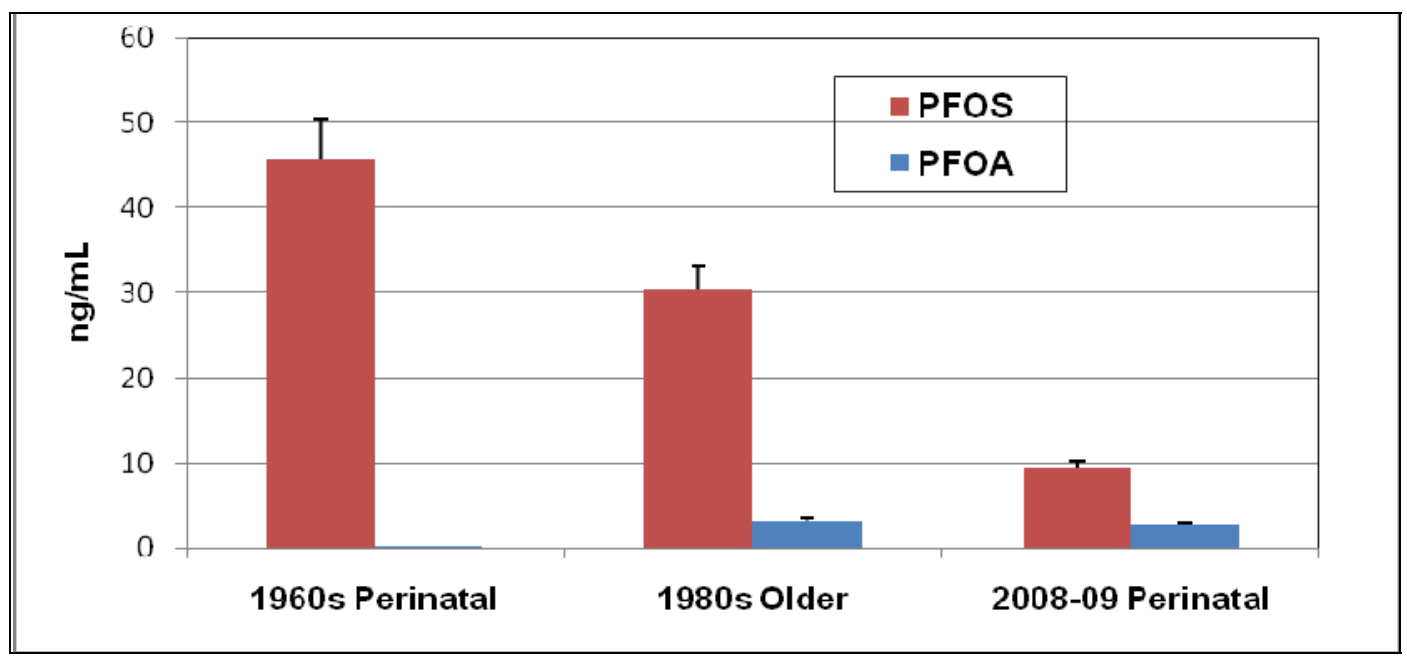

Figure 3. Perfluorinated chemicals in Serum

\section{CONCLUSIONS}

Californians continue to have high PBDE levels, exceeding US averages (NHANES). PBDEs were not measurable in the 1960s, or 1980s, and were first observed in the mid- to late-1990s. PFOS levels were surprisingly high in the 1960s, a period not well studied before, and have been declining since then. On the other hand, PFOA appears to have peaked in the 1980s. As more samples are collected, work is under way to explore determinants of exposures to PBDEs and new BFRs, and whether the lower levels observed in contemporary adult women are a reflection of age, or a result of the recent ban of Penta- and Octa-BDEs. Similarly, predictors of exposures to PCBs, OCPs and PFCs are being investigated.

\section{ACKNOWLEDGEMENTS AND DISCLAIMER}

We thank all the participants of the original studies who provided samples. We also thank Dr. Wiemels of the University of California San Francisco, and Dr. Cohn of the California Health and Development Studies for access to their archived serum samples. The ideas and opinions expressed herein are those of the authors and do not necessarily reflect the official position of the California Department of Toxic Substances Control.

\section{REFERENCES}

Brown F.R., Winkler J., Visita P., Park J.S., Petreas M., (2007) Simultaneous Analysis of PCBs and Polybrominated Diphenyl Ethers (PBDEs) in Human Adipose Using GC/MS/MS, Organohalogen Compounds, 69, 1199-1202.

California Environmental Contaminant Biomonitoring Program (CECBP). www.oehha.ca.gov/Biomonitoring

Hooper K., She J., Sharp M., Jewell N., Gephart R., Chow J., Holden A., (2007) Depuration of Polybrominated Diphenyl Ethers (PBDEs) and Polychlorinated Biphenyls (PCBs) in Breast Milk from California First-Time Mothers (Primiparae), Environ Health Perspect, 115, 1271-1275.

National Health and Nutrition Examination Survey http://www.cdc.gov/exposurereport/pdf/FourthReport.pdf

Petreas M., She J., Brown F., Winkler J., Windham G., Rogers E., Zhao G., Bhatia R., Charles M., (2003) High body burdens of 22'44'-brominated diphenyl ether in California women, Environmental Health Perspectives, 111, 1175-1179.

Petreas M., Nelson D., Brown F.R., Goldberg D., Hurley S., Reynolds P., (2011) High concentrations of polybrominated diphenylethers (PBDEs) in breast adipose tissue of California women, Environment International., 37, 190-197.

Rogers E., Petreas M., Park J., Zhao G., Charles M., (2004) Evaluation of Four Capillary Columns for the Analysis of Organochlorine Pesticides (OCPs), Polychlorinated Biphenyls (PCBs), and Polybrominated Diphenyl Ethers (BDEs) in Human Serum for Epidemiologic Studies, Journal of Chromatography B, 813, 269-285. 
She J., Holden A., Sharp M., Tanner M., Williams-Derry C., Hooper K., (2007) Polybrominated Diphenyl Ethers (PBDEs) and Polychlorinated Biphenyls (PCBs) in Breast Milk from the Pacific Northwest, Chemospher,. 67, S307-317.

Sjodin A., Wong L., Jones R., Park A., Zhang Y., Hodge C., DiPietro E., McClure C., Turner W., Needham L., Patterson D., (2008) Serum Concentrations of Polybrominated Diphenyl Ethers (PBDEs) and Polybrominated Biphenyl (PBB) in the United States Population: 2003-2004, Envir Sci Technol, 42, 1377-1384.

Wang Y., Park J-S., Guo W., Zota A., Petreas M., (2010) Analysis of PBDEs, PCBs, organochlorine pesticides, and new BFR alternatives in California pregnant women by high-resolution mass spectrometry, Organohalogen Compounds, 72, 181-184.

Wang M., Park J-S., Petreas M. (2011) Temporal changes in the levels of perfluorinated compounds in California women serum over the past 50 Years, Envir Sci Technol, 45, 7510-7516.

Windham G., Lee D., Mitchell P., Anderson M., Petreas M., Lasley B., (2005) Exposure to Organochlorine Compounds and Effects on Ovarian Function, Epidemiology, 16, 182-190.

Zota A., Schwartz J.M., Steinauer J., Petreas M., Pasternack T., Woodruff T., (2010) Impact of Polybrominated Diphenyl Ethers on Thyroid Hormone Levels Among California Women During Second Trimester of Pregnancy. Joint ISES-ISEE Conference, Seoul, Korea. 